\title{
Structurally isolated photoactuation of graphene-mixed temperature-responsive hydrogels in soft-rigid series structure
}

\author{
Tomoki Watanabe ${ }^{1 \dagger}$, Yoshiyuki Yokoyama ${ }^{2}$ and Takeshi Hayakawa ${ }^{1{ }^{*}+}$ (D)
}

\begin{abstract}
This paper presents fabrication and actuation methods for a soft microrobot with a hybrid structure composed of soft microactuators and a rigid supporting body. This hybrid structure enables actuation of the microrobot with independent driving of multiple actuators to provide complex movement like that of living microorganisms. We use the temperature-responsive hydrogel poly(N-isopropylacrylamide) (PNIPAAm) as a soft microactuator. PNIPAAm swells with water at low temperature but shrinks at high temperature. This volume change thus allows PNIPAAm to be used as an actuator by controlling its temperature. We successfully fabricated the microrobot with its soft-rigid hybrid structure composed of PNIPAAm and rigid photoresist using a multilayered microfabrication process. In addition, we used a sacrificial layer process to release the fabricated microrobot from the substrate to allow it to move freely. To actuate the microrobot, we mixed PNIPAAm with graphene, which has a high photothermal conversion efficiency. The temperature of the soft actuator when mixed with graphene can be increased by irradiating it with light. Therefore, actuation of the microrobot is achieved by sequentially irradiating the microactuators with focused light. We present the fabrication, release and partial actuation of the microrobot to demonstrate the feasibility of the proposed microrobot with the soft-rigid hybrid structure in this paper.
\end{abstract}

Keywords: Soft robot, Gel actuator, Microrobot, PNIPAAm, Graphene

\section{Introduction}

In recent decades, soft robots have been attracting considerable attention because these robots have potential to bring a new era of robotics and be used in numerous practical applications [1-9]. From the viewpoint of basic research, artificial mechanisms with softness similar to that of living organisms can aid in understanding the origins of unique functions or the high efficiency of the mechanisms of living organisms. By understanding these origins, we can realize robots that have new functions or greater efficiency by correctly mimicking the mechanisms of living organisms, such as the locomotion

\footnotetext{
*Correspondence: hayaka-t@mech.chuo-u.ac.jp

†Tomoki Watanabe and Takeshi Hayakawa contributed equally to this work

${ }^{1}$ Department of Precision Engineering, Chuo University, 1-13-27 Kasuga, Bunkyo-ku, Tokyo 112-8551, Japan

Full list of author information is available at the end of the article
}

of humans $[4,5]$, the swimming mechanisms of fish [6] or the peristaltic motion of earthworms [7]. In addition to basic research, soft robots could also possibly be used in a number of practical applications. For example, their softness would allow cooperative working involving robots and humans to be realized with reduced risk of damage or harm in the case of an accident [8]. Another potential application of soft robots is to act as a flexible soft gripper [9]. A soft gripper can be used to grip target objects with wide size, shape and stiffness ranges because the gripper can change its shape according to the target object.

When focusing on the mechanisms of motion of living organisms, one of the most important points is their use of hybrid structures composed of soft structural components and a rigid supporting body. For example, mammals, birds and fish all have hybrid structures composed of soft muscles and rigid skeletons. In addition, insects 
have soft muscles located inside rigid exoskeletons. These hybrid structures work excellently and can be used to realize a variety of movements, including walking, running, swimming or flying. Therefore, these soft-rigid hybrid structures are thought to be a key feature of living organisms. In fact, most reported soft robots have hybrid structures comprising soft actuators and rigid supporting bodies [4-9].

As we noted above, such soft robots have major potential and have been intensively researched. However, most of these reported soft robots are on the macroscale, i.e., they have sizes ranging from the millimeter scale to the meter scale. On the microscale, living microorganisms also have hybrid structures composed of soft actuators and rigid supporting bodies. For example, some bacteria have rigid cell walls that act as a supporting body and a flagellum that acts as a soft actuator to enable swimming in water. A paramecium does not have a cell wall but it does have a cytoskeletal structure that acts as a supporting body and numerous cilia that act as soft actuators on the surface of their cell membrane. The cilia on the paramecium move sequentially and realize peristaltic motion to generate sufficient flow to swim. Human body cells also have cytoskeletal supporting bodies and actin fibers that act as soft actuators inside their cell membranes. Therefore, even on the microscale, hybrid structures of soft actuators and rigid supporting bodies appear to be essential to the movements and functions of living microorganisms.

Some soft microrobots on the $\mu \mathrm{m}$ to $\mathrm{mm}$ scale have also been reported [10-18]. For example, Palagi et al. fabricated soft liquid-crystal elastomer (LCE) actuators and moved them via light irradiation [16]. However, this robot was constructed using soft structures only. It is difficult to realize soft-rigid hybrid structures that mimic living microorganisms. Nawroth et al. and Akiyama et al. succeeded in fabricating a hybrid structure composed of living cells and a rigid supporting body $[17,18]$. They used rat cardiomyocytes or mammalian muscle cells as the soft actuators and placed them on rigid microstructures. They also successfully actuated the muscle cells by application of an external electric field or light irradiation and thus moved the robots. However, these soft actuators were made from living cells and thus degraded in a short time and might have low repeatability. Therefore, in the case of using the microrobot for long term, such as cell manipulation in long term cell culturing devices or in-vivo study, it is important to realize a soft microrobot with a soft-rigid hybrid structure that is made completely from artificial materials.

Another important point with regard to microrobots is the number of integrated actuators to the robots. In conventional works, relatively simple structures have been used, such as structures composed of soft materials only [16], stacked sheets of rigid photoresist and muscle cells [17], or muscle cells placed on a rigid microstructure [18]. These robots are actuated by light irradiation or application of an electric field to the robot. These spatially uniform external fields actuate all parts of the soft actuators on the microrobots uniformly. Conventional structures and actuation methods can thus be used to achieve relatively simple motions that cannot independently drive multiple actuators, such as thrusting motion or walking in one direction, as shown in Fig. 1a. To realize more complex motion like that of living microorganisms, independent actuation of multiple soft actuators on the microrobots is necessary. By actuating multiple actuators independently, soft microrobots can realize complex motion like that of living microorganisms, e.g., swimming, turning or peristaltic motion, as shown in Fig. 1b. Therefore, the two important techniques required to realize soft microrobots that can mimic living microorganisms are (i) a fabrication method for a soft-rigid hybrid structure made from artificial materials, and (ii) a method for independent actuation of the multiple actuators on these robots.

In this paper, we propose a soft microrobot with a softrigid hybrid structure. The proposed robot is fabricated using a microfabrication process and is made entirely from artificial materials. The proposed hybrid robot can be actuated via light irradiation and this will enable independent actuation of multiple actuators on the microrobot. Next, we present the concept of the soft microrobot and explain the actuation mechanism. Subsequently, we perform experimental and theoretical evaluations of the soft actuator and describe the fabrication method of the
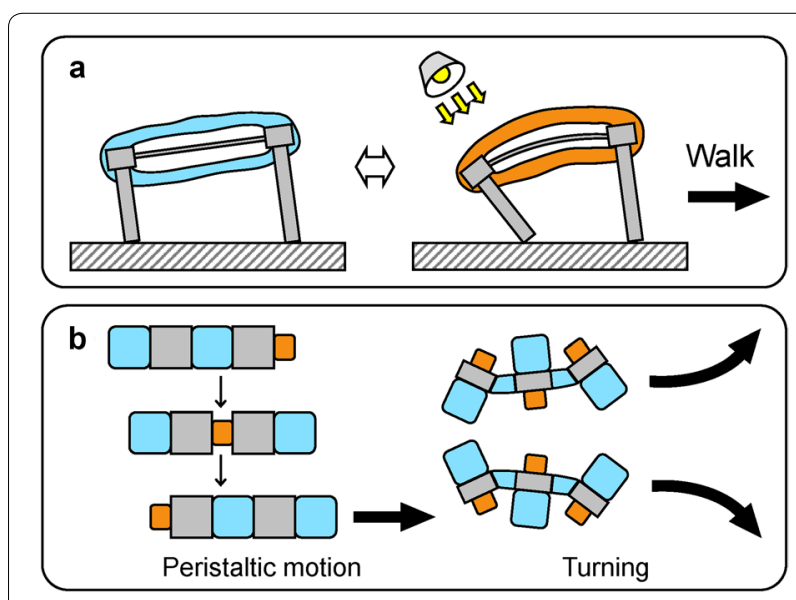

Non driven actuator $\square$ Rigid structure $\square$ Driven actuator

Fig. 1 Motion of soft microrobot. a Motion with actuation of single soft actuator, and $\mathbf{b}$ independent actuation of multiple soft actuators 
proposed soft microrobot. Finally, we fabricate the robot and demonstrate the feasibility of the proposed actuation method for this robot.

\section{Concept}

To realize a microrobot with soft-rigid hybrid structures, we propose a soft microrobot made from temperatureresponsive gel and photoresist material, as shown in Fig. 2. The proposed soft microrobot has bodies composed of temperature-responsive gels that operate as soft actuators and photoresist acting as the rigid supporting body. For the soft actuators, we use the photo-patternable temperature-responsive gel poly( $\mathrm{N}$-isopropylacrylamide) (PNIPAAm; Bioresist, Nissan Chemical Corp., Tokyo, Japan) [19]. PNIPAAm is a hydrogel that contains water as part of a solution. It swells with environmental water content at low temperatures $\left(\leq 32{ }^{\circ} \mathrm{C}\right)$ and shrinks at high temperatures $\left(\geq 32{ }^{\circ} \mathrm{C}\right)$. Bioresist is photo-patternable PNIPAAm, which can be patterned on a substrate with fixed structure by using UV exposure. Therefore, we can use volume changes of patterned bioresist on a substrate as an actuator, as shown in the inset of Fig. 2 [20-22]. Therefore, we can use this volume change to provide the displacement of the actuator by controlling the temperature of the PNIPAAm. The rigid supporting body is made from photoresist (SU-8, Kayaku Microchem Co. Ltd., Tokyo, Japan). These soft actuators and rigid supporting bodies can be fabricated via a multilayered photolithography process with accuracy of the $\mu \mathrm{m}$ order. While usual microfabricated structures are fixed to the substrate, the fabricated microrobot must be released from the substrate. Therefore, we use a sacrificial layer process based on use of dextran [23,24], as will be described in detail later in the paper.

To actuate the robot, we use light irradiation to heat the soft actuator locally. Normally, actuation of microfabricated PNIPAAm structures is performed using a microheater [25-27]. However, the wiring of such a microheater would prevent free movement of the microrobot. Therefore, we use light irradiation-based temperature control to actuate the microrobot. The main constituent of the PNIPAAm solution is water and its light absorption is low. Therefore, we mixed PNIPAAm with graphene, which is known to be a high-efficiency photothermal conversion material [28-30]. Other candidates for light absorber are metal nanomaterials, such as gold or silver nanoparticles. These materials have higher wavelength selectivity for light absorption in UV to visible light region. On the other hand, carbon nanomaterials, such as graphene, or carbon nanotubes have wider absorption spectrum from UV to IR region. [28, 31] In this study, we employed graphene which has high absorption at IR region because we used IR laser for driving of the actuator. The temperature of PNIPAAm when mixed with graphene can be increased by irradiating it using light. In addition, we can selectively actuate one actuator among multiple actuators by irradiating it with focused light, as shown in Fig. 2. Therefore, independent actuation of the multiple actuators on the soft microrobot can be achieved by irradiating the patterned soft actuators sequentially with focused light. The proposed actuation method will allow independent actuation of the multiple

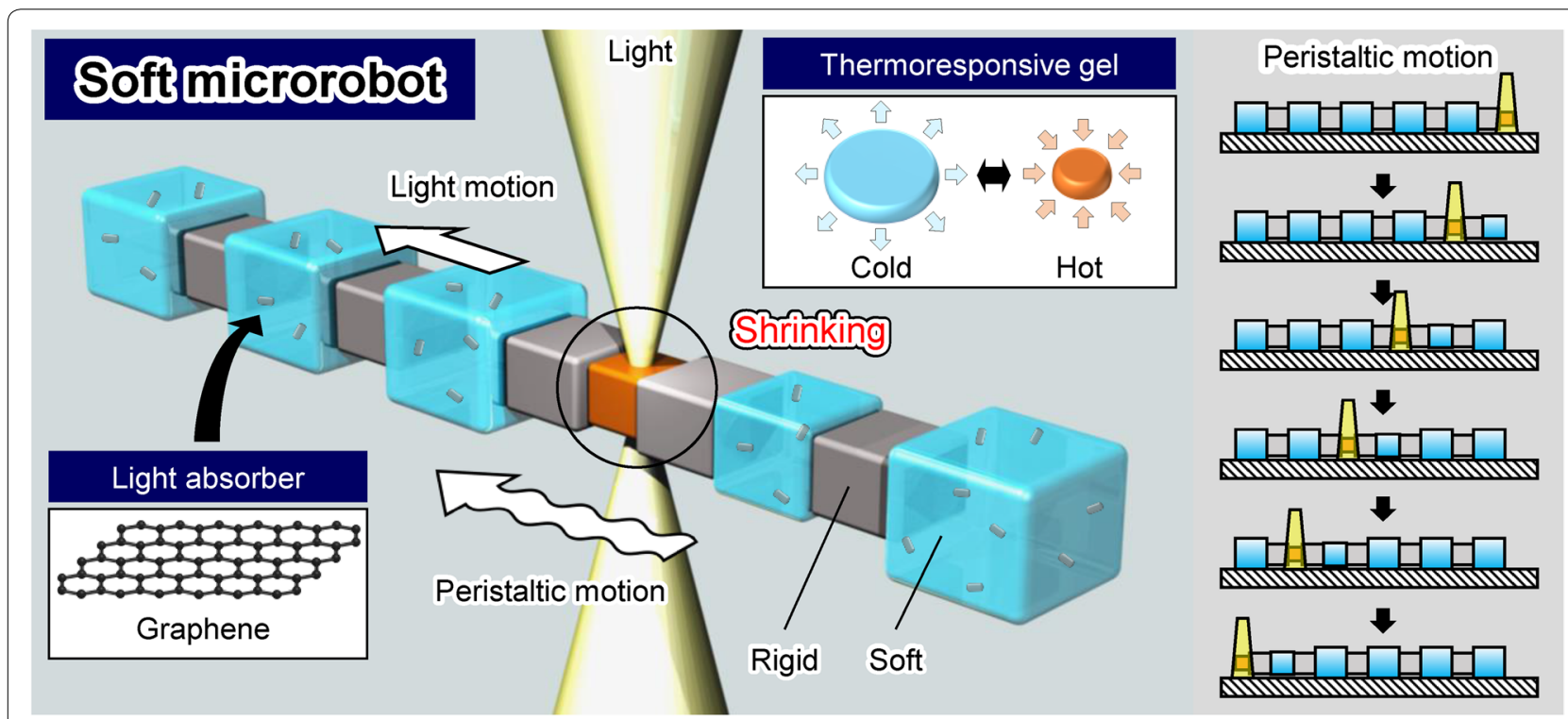

Fig. 2 Concept of soft microrobot having with soft-rigid hybrid structures 
actuators on the microrobot to realize complex movements such as the peristaltic, turning, or swimming motions of living microorganisms.

In this study, we fabricated a straight-structured microrobot with soft-rigid hybrid structures containing six actuators, as shown in Fig. 2.

\section{Light driving of soft actuator}

First, we evaluated the driving characteristics of patterned PNIPAAm acting as a soft actuator when driven using the proposed method.

\section{Experiments}

We fabricated patterns of various sizes using PNIPAAm mixed with graphene on a glass substrate. These PNIPAAm patterns were fabricated via standard photolithography processes. We mixed graphene ink (793663-5ML, Sigma-Aldrich Japan, Tokyo, Japan) into Bioresist up to $3.7 \%$ of its volume. We fabricated circular-shaped patterns of PNIPAAm with diameters of $200 \mu \mathrm{m}$ and $40 \mu \mathrm{m}$ to confirm the differences in the drive characteristics that occur according to the actuator sizes.

The experimental system used to irradiate the actuators with light is shown in Fig. 3. To drive the soft actuators, we focused an infrared (IR) laser (LuxX 1060-150, Omicron-Laserage Laserprodukte $\mathrm{GmbH}$, RodgauDudenhofen, Germany; wavelength: $1060 \mathrm{~nm}$ ) with $150 \mathrm{~mW}$ output power using an objective lens (Plan N 10x/0.25na, Olympus, Tokyo, Japan). The power distribution of this laser is gaussian and its full width at half maximum (FWHM) is $750 \mu \mathrm{m}$. The microscopic images were observed and recorded using a charge-coupled device (CCD) camera (BFS-U3-32S4, FLIR Systems Japan K.K., Tokyo, Japan). To evaluate the frequency responses of the actuators, we input square waves with frequencies of $0.01-5.00 \mathrm{~Hz}$ to the laser controller. Using this input

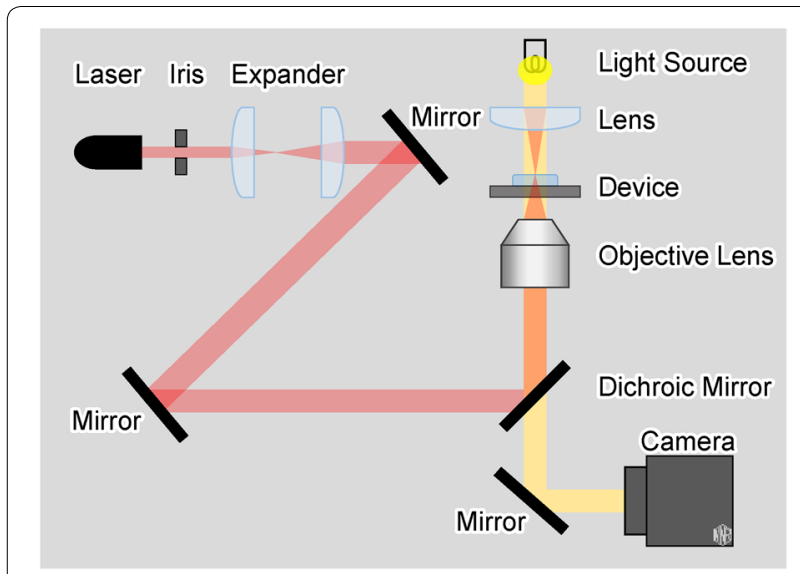

Fig. 3 Experimental system signal, the IR laser was switched on and off at these input frequencies.

\section{Evaluation of drive characteristics of soft actuator}

First, we succeeded in driving the patterned soft actuator by irradiating it with the focused IR laser beam, as shown in Fig. 4a. To evaluate the displacements of the actuator, we define the displacement as follows.

$$
\text { Displacement ratio : } \frac{D_{\text {diff }}}{D_{M A X}-D_{M I N}}
$$

Here, $D_{\text {diff }}$ is the difference in the diameter of the actuator before and after laser irradiation, and $D_{M A X}$ and $D_{M I N}$ are the maximum and minimum values of the diameters, respectively.

The measured displacement at each frequency is shown in Fig. 4b. From Fig. 4b, the displacements of the actuators of both sizes were reduced by increasing the operating frequency. From Fig. 4b, the displacements of the 40 $\mu \mathrm{m}$ actuator are larger than those of the $200 \mu \mathrm{m}$ actuator. These results show that the PNIPAAm patterns with smaller sizes produce faster response characteristics. This is thought to occur because of the scale effect of the thermal transfer inside the actuator. Therefore, we estimate the scale effect theoretically in the next subsection.

\section{Theoretical analysis of the scale effect on thermal transfer}

To estimate the scale effects on the response characteristics, we performed a theoretical analysis of the thermal transfer in the actuator. The model for this analysis is shown in Fig. 5. We assume that the temperature at the

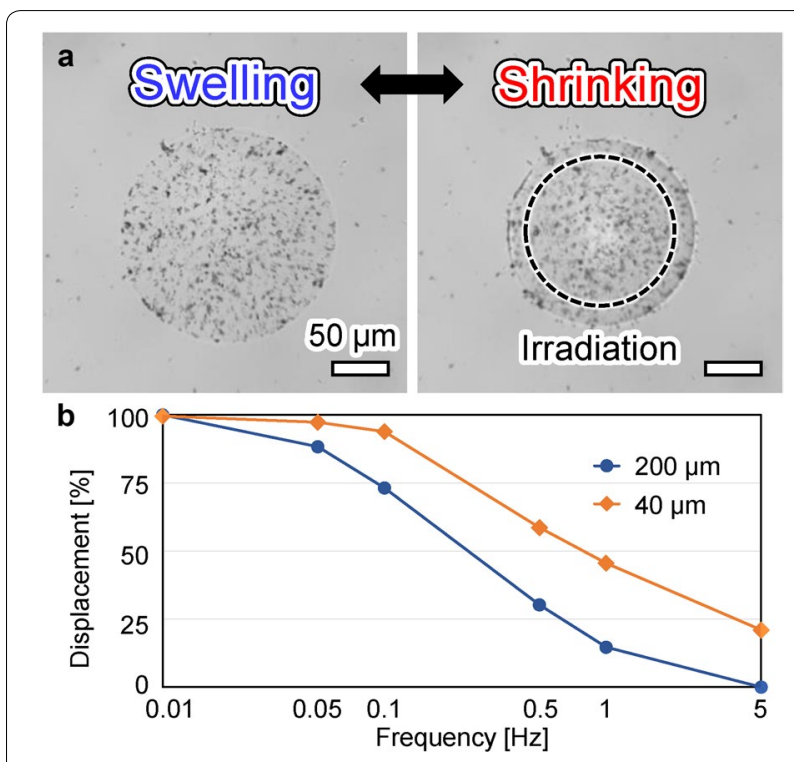

Fig. 4 Driving of the soft actuator. a Microscopic images, and $\mathbf{b}$ frequency response 

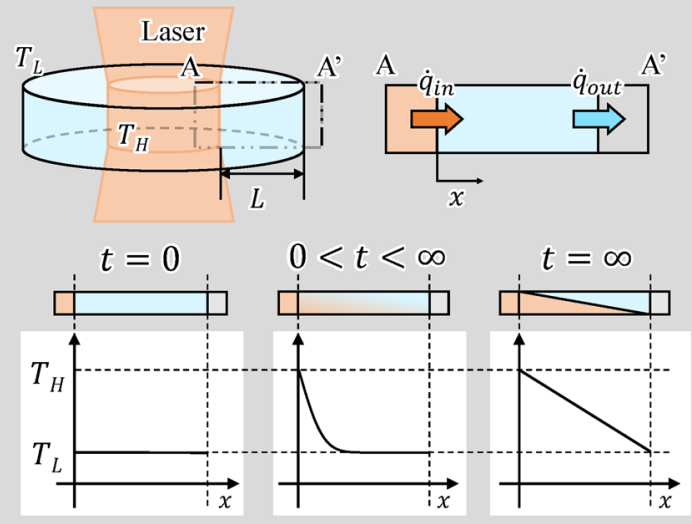

Fig. 5 Theoretical analysis model

laser-irradiated point increases much more quickly than the temperature increases at any other parts of the actuator. Therefore, we set the temperature of the laser-irradiated point to be the constant high temperature $T_{H}$. In addition, we assume that the environmental temperature is also constant because the volume of water in the environment is much larger than that in the patterned actuator. Therefore, we set the environmental temperature to be the constant low temperature $T_{L}$. Under these assumptions, when the laser irradiates the center of the circular-shaped actuators, the thermal transfer phenomenon can be described using a one-dimensional equation along the radial direction shown as the $x$ axis $\left(\mathrm{A}-\mathrm{A}^{\prime}\right)$ in Fig. 5.

We used a Fourier equation that describes the nonequilibrium heat conduction to evaluate the transient phenomenon of temperature change that occurs when the laser is irradiating the microactuator. The one-dimensional Fourier equation is written as follows:

$$
\frac{\partial T}{\partial t}=\alpha \frac{\partial^{2} T}{\partial^{2} x}
$$

where $T$ is the temperature at coordinate $x$ and time $t$, and $\alpha$ is the thermal diffusivity, which is described as $\alpha=\kappa / \rho c$. Here, $\kappa, \rho$, and $c$ are material constants, i.e., the thermal conductivity, the density and the specific heat, respectively. For reference, the value of $\alpha$ of water is $1.44 \times 10^{-7}\left[\mathrm{~m}^{2} / \mathrm{s}\right]$ and SU-8 (epoxy) is $1.47 \times 10^{-7}\left[\mathrm{~m}^{2} / \mathrm{s}\right]$ at $20^{\circ} \mathrm{C}$, respectively.

This equation can be solved using Laplace and inverse Laplace transformations under the following boundary and initial conditions.

$$
\begin{aligned}
& T(x, t=0)=T_{L} \\
& T(x=0, t)=T_{H} \\
& T(x=L, t)=T_{L}
\end{aligned}
$$

The derived solution to Eq. (2) can be written as a temperature difference using a polynomial form, as follows.

$$
\begin{aligned}
\frac{T(x, t)-T_{L}}{T_{H}-T_{L}}= & 1-\frac{x}{L} \\
& -\sum_{n=1}^{\infty} \frac{2}{\pi n} \exp \left(-\frac{\pi^{2} n^{2} \alpha}{L^{2}} t\right) \sin \left(\pi n \frac{x}{L}\right)
\end{aligned}
$$

This equation (6) can be rewritten in a dimensionless form by introducing the dimensionless variables $\hat{x}=x / L$ and $\hat{t}=\alpha t / L^{2}$. Using dimensionless Eq. (6) and following Fourier's law,

$$
\dot{q}=-\kappa \frac{\partial T}{\partial x}
$$

the dimensionless heat flux density $\dot{q}$ at the two sides of the model, given by $\dot{q}_{\text {in }}(x=0, t)$ and $\dot{q}_{\text {out }}(x=L, t)$ as shown in Fig. 5, can be calculated as follows.

$$
\begin{aligned}
& \dot{q}_{\text {in }}(x=0, t)=1+2 \sum_{n=1}^{\infty} \exp \left(-\frac{\pi^{2} n^{2} \alpha}{L^{2}} t\right) \\
& \dot{q}_{\text {out }}(x=L, t)=1+2 \sum_{n=1}^{\infty}(-1)^{n} \exp \left(-\frac{\pi^{2} n^{2} \alpha}{L^{2}} t\right)
\end{aligned}
$$

After sufficient time passes to allow the system to reach a steady state, the exponential terms of Eqs. (8) and (9) become 0 and $\dot{q}_{\text {in }}(x=0, t=\infty)=\dot{q}_{\text {out }}(x=L, t=\infty)=1$, respectively. We then estimate the relaxation time $t_{r}$, which is the time required for the system to reach a steady state, as follows.

$$
2 \exp \left(-\frac{\pi^{2} \alpha}{L^{2}} t_{r}\right) \approx 0.01
$$

This equation is calculated under assumption that the exponential terms are 100 times smaller than 1. $t_{r}$ can then be written as follows.

$$
t_{r}=\ln \left(\frac{0.005}{\pi^{2}}\right) \frac{L^{2}}{\alpha}=0.054 \frac{L^{2}}{\alpha}
$$

The response time of the actuator should be proportional to the relaxation time $t_{r}$ because the actuator is driven according to its temperature change. Eq. (11) indicates that $t_{r}$ is proportional to $L^{2}$. Therefore, an actuator of smaller size will have better response characteristics, as we experimentally evaluated in Fig. 4. For reference, the distribution of displacement of the actuator can be experimentally observed in Additional file 1, section "Frequency response". In this video, central part of the actuator with size of $200 \mu \mathrm{m}$ firstly shrank. After that, 
edge part of the actuator shrank as our assumption in the theoretical analysis. While we performed the theoretical analysis for the heating process based on light irradiation, a similar scale effect should be confirmed for the cooling process produced by turning the laser off.

\section{Fabrication and actuation of soft microrobot}

Next, we evaluate the process conditions for the proposed sacrificial layer process using dextran.

\section{Evaluation of sacrificial layer process conditions}

The fabrication process for the soft microrobot is illustrated in Fig. 6. Basically, the proposed microrobot is fabricated using multilayered photolithography processes (Fig. 6b-e). However, to allow the microrobot to move freely after fabrication, the microrobot must be released from the substrate. Therefore, we first fabricate a sacrificial layer on the Si substrate, as shown in Fig. 6a. Subsequently, the microrobots are patterned on the sacrificial layer. To prevent the sacrificial layer from being dissolved in the organic solvents that are used to develop the SU-8 and Bioresist (Fig. $6 \mathrm{c}$ and e), the materials used in the sacrificial layer should be insoluble in the relevant organic solvents. In this study, we used dextran as the sacrificial layer material. Dextran is a type of polysaccharide that is insoluble in organic solvents under appropriate processing conditions [23, 24]. In contrast, the water solubility of dextran is very high. Therefore, it is possible to release the microrobot from the substrate using water after the process in which SU-8 and Bioresist are patterned using organic solvents, as shown in Fig. $6 \mathrm{f}-\mathrm{h}$. For exposure of bioresist, normal mask aligner (PEM-800, Union Optical Co., Ltd., Tokyo, Japan) with a mercury lamp was used. Bioresist is mainly exposed with $365 \mathrm{~nm}$ (i-line) UV light similar to common photoresists. In this study, we

a

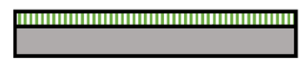

b

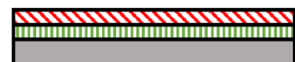

C

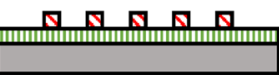

d

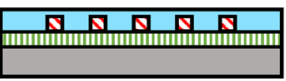

Si 四 Dextran $\mathbb{Q}$ SU-8

Fig. 6 Soft microrobot fabrication process expose the bioresists for $400 \mathrm{~s}$ without an exposure filter. Detailed evaluations of pattern resolution for bioresists were previously evaluated by Ito et al. [27].

First, we confirm the process conditions for the dextran sacrificial layer. The dextran is dissolved in water and spin-coated on a Si substrate. After that, the substrate is baked at high temperature. Therefore, the process conditions to be determined are (i) the solution concentration, (ii) the spin coating conditions and (iii) the bake temperature. We fabricated the dextran layer under varying solution concentration conditions of 17, 33 and 43 wt.\%. Similarly, the spin coating rotation speeds were varied as 500, 2000 and $6000 \mathrm{rpm}$ and the bake temperatures were varied as 60,120 and $180{ }^{\circ} \mathrm{C}$. After the dextran layer is fabricated under various process conditions, we dip the substrate into ethanol at room temperature and confirm that the dextran layer is insoluble in ethanol. Additionally, we measured the time required for the dextran to dissolve in the water for each process condition.

A summary of the evaluation of the dextran solution concentration is shown in Table 1. These results show that the dextran solution concentration strongly affects the solubility of the dextran layer in ethanol. When the solution concentration was low, the dextran layer was dissolved in ethanol. However, when the solution concentration was high, the dextran layer was not dissolved in ethanol. Therefore, we decided to use the condition that the solution concentration is $43 \mathrm{wt} . \%$. In addition, the spin coating rotation and bake temperature conditions do not affect the solubility of dextran with respect to ethanol. These conditions only affect the times required for the layer to dissolve in water. Therefore, we decided on a spin-coating rotation speed of $6000 \mathrm{rpm}$ and a bake temperature of $180^{\circ} \mathrm{C}$.

\section{Fabrication of the soft microrobot}

According to the results of evaluation of the sacrificial layer process in the previous subsection, we fabricated the microrobot using the fabrication process shown in Fig. 6. As a design for the microrobot, five rigid supporting bodies of $60 \mu \mathrm{m}$ square in size and six soft actuators of $100 \mu \mathrm{m}$ square in size are connected in a straight line, as shown in Fig. 7a. We successfully fabricated the softrigid hybrid structure, as shown in Fig. 7a.

Table 1 Processing conditions for sacrificial layer

\begin{tabular}{lll}
\hline $\begin{array}{l}\text { Solution } \\
\text { concentration (wt.\%) }\end{array}$ & Solubility in ethanol & $\begin{array}{l}\text { Time } \\
\text { to dissolve } \\
\text { in water (s) }\end{array}$ \\
\hline 17 & Slightly dissolved & $5-10$ \\
33 & Slightly dissolved & 20 \\
43 & Unchanged & 50 \\
\hline
\end{tabular}



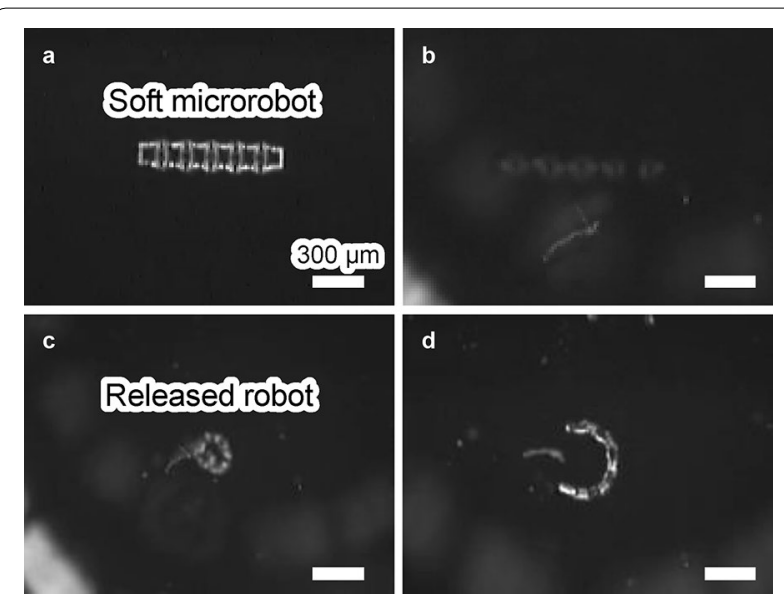

Fig. 7 Sacrificial process for release of soft microrobot. a Before immersion in water, $\mathbf{b}$ immediately after immersion in water, and c 20 $\mathrm{s}$ and $\mathbf{d} 40 \mathrm{~s}$ after immersion

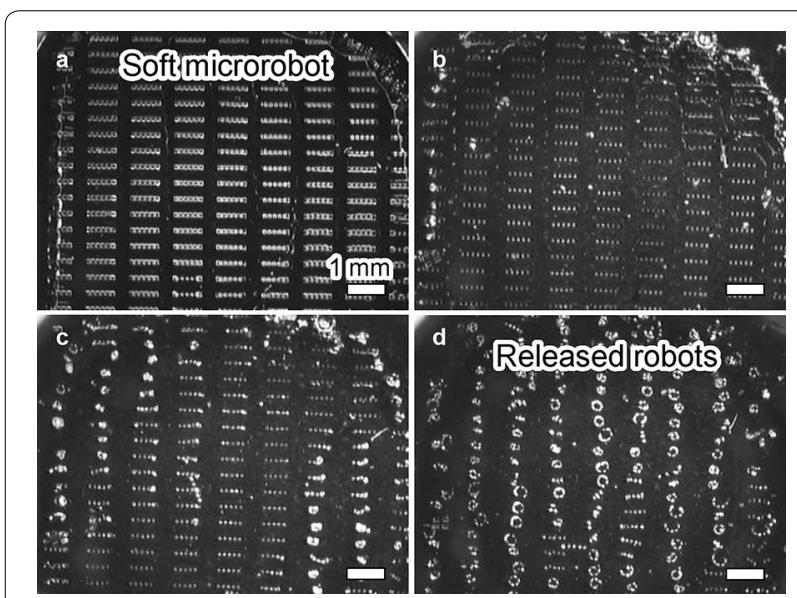

Fig. 8 Mass production and release of soft microrobot. a Before immersion in water, and $\mathbf{b}$ - $\mathbf{d}$ after immersion in water
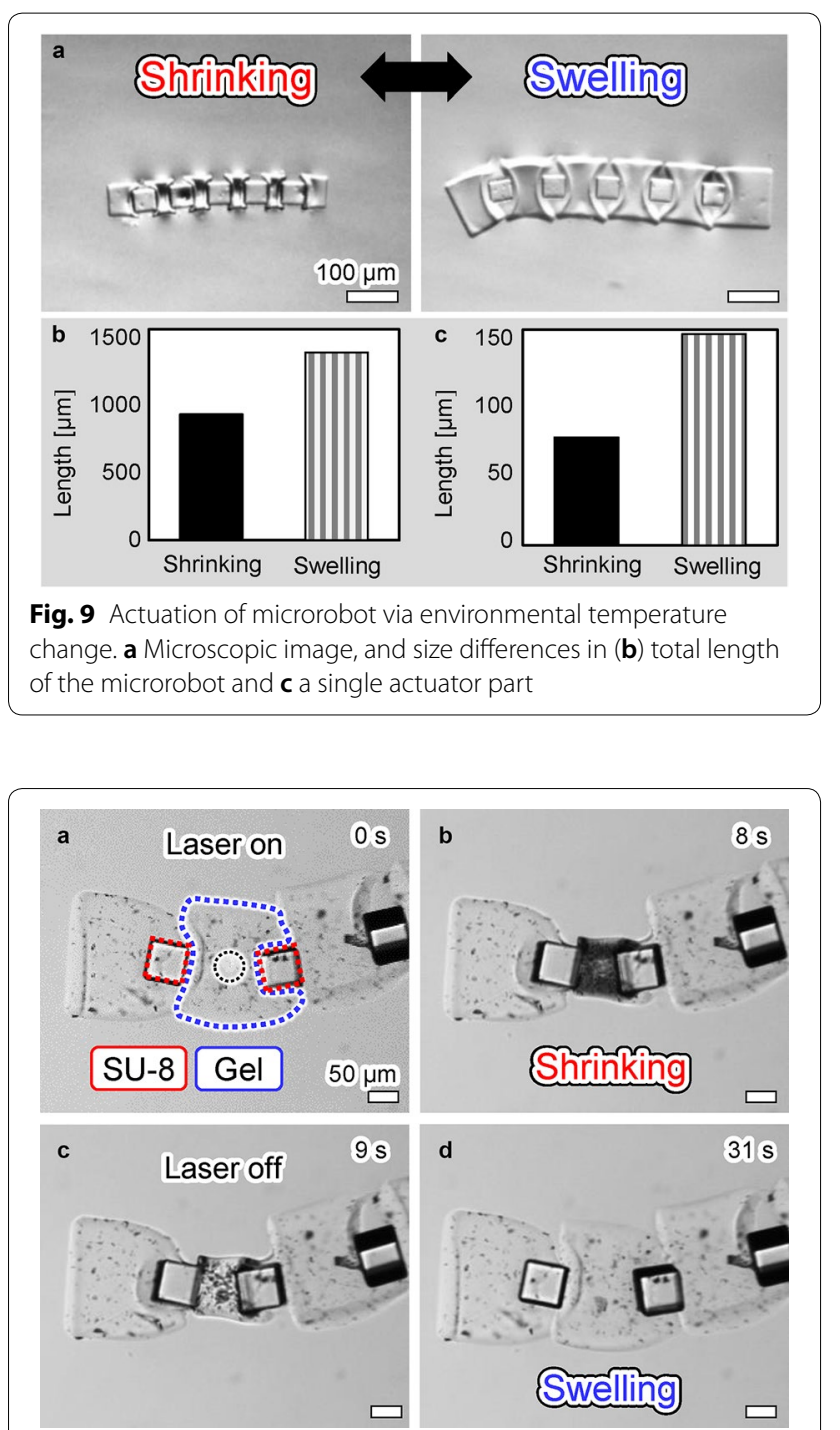

Fig. 10 Actuation of the soft microrobot. a Start of laser irradiation; $\mathbf{b}$ $8 \mathrm{~s}$ after laser irradiation begins; c laser irradiation stops; and (d) $22 \mathrm{~s}$ after the laser irradiation stopped
Then, we immersed the substrate in water and confirmed that the microrobot was released, as shown in Fig. $7 \mathrm{~b}-\mathrm{d}$. The fabricated microrobots were rolled immediately after the release process, as shown in Fig. 7c. After $40 \mathrm{~s}$, the soft actuators of the robot swell with water and the robots become straight, as shown in Fig. $7 \mathrm{~d}$. In addition, we tried to fabricate large numbers of microrobots, as shown in Fig. 8. All the patterned microrobots were released successfully from the substrate.

\section{Actuation of soft microrobot by light irradiation}

Here, we actuated the fabricated soft microrobot using the proposed actuation method. First, we confirmed size change in the robot by varying the temperature of the surrounding water, as shown in Fig. 9.
The fabricated robots were placed on a glass substrate with surrounding water. After that, the glass substrate was heated using a glass heater (KM-1, AS ONE Corporation, Osaka, Japan). The fabricated robot successfully swelled at room temperature and shrunk at higher temperatures $\left(\geq 50{ }^{\circ} \mathrm{C}\right)$, as shown in Fig. 9a. The overall length of the microrobot in the swollen state becomes 1.5 times longer than that in the shrunken state, as shown in Fig. 9b. In addition, the length of each soft actuator doubled in the swollen state, as shown in Fig. 9c. Furthermore, we confirmed that the soft actuator can be actuated by IR laser irradiation, as shown in Fig. 10. Under IR laser irradiation, the soft actuator shrinks within $8 \mathrm{~s}$, as shown in Fig. 10b. In addition, the 

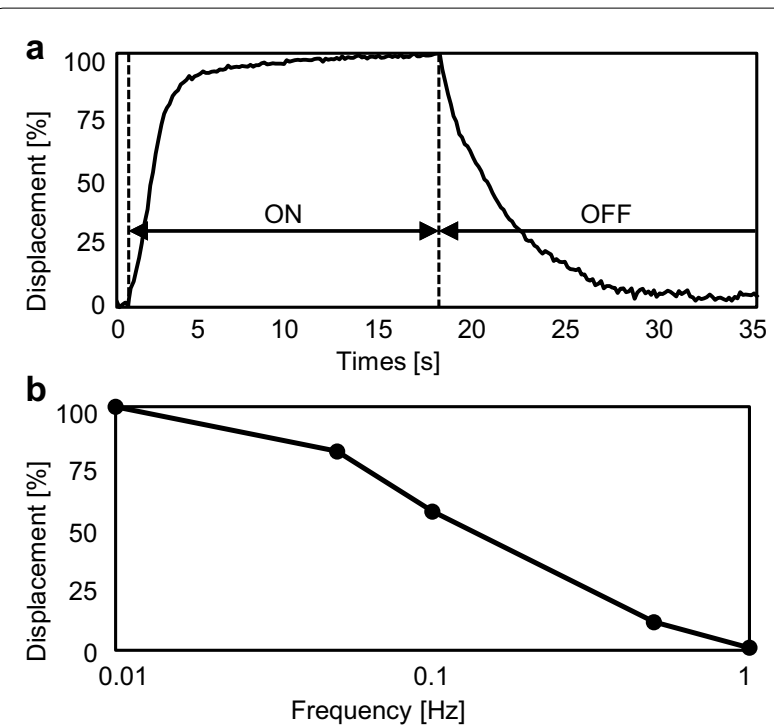

Fig. 11 Drive characteristics of the soft microrobot. a Step response, and $\mathbf{b}$ frequency response

actuator swells to its original volume within approximately $20 \mathrm{~s}$ of the irradiation stopping, as shown in Fig. 10c, d.

Finally, we evaluated the step and frequency responses of the soft actuators on the microrobot, as shown in Fig. 11a, b. Figure 11a shows that the soft actuator shrinks approximately $5 \mathrm{~s}$ after laser irradiation starts and swells approximately $15 \mathrm{~s}$ after the irradiation stops. Figure $11 \mathrm{~b}$ shows the frequency response over the range from 0.01 to $1.00 \mathrm{~Hz}$. From Fig. 11b, we confirmed that the soft actuator can be driven at a frequency of at least $0.1 \mathrm{~Hz}$ with approximately $50 \%$ displacement relative to the maximum. These response characteristics can be improved by making the microrobot smaller or by changing the light absorbing materials or the mixing ratio of the materials.

\section{Conclusions}

In this study, we proposed a soft microrobot with a soft-rigid hybrid structure. The proposed robot consists of micropatterned soft actuators made from a temperature-responsive gel and a rigid photoresist. We mixed the soft actuator with graphene to act as a light absorber and we can control the temperature of the actuator by irradiating it with light. Therefore, the proposed microrobot can be actuated by sequentially irradiating its actuators using focused light. First, we evaluated the basic drive characteristics of the actuator and theoretically analyzed the thermal transfer within the actuator. After that, we fabricated the proposed microrobot with the soft-rigid hybrid structure using a sacrificial layer process. In addition, we succeeded in partially actuating the actuator on the microrobot by irradiating it with focused light and evaluated its driving characteristics. The construction of a suitable light irradiation system and realization of complex actuator motion such as that of living microorganisms will be reported in future work.

\section{Supplementary information}

Supplementary information accompanies this paper at https://doi. org/10.1186/s40648-019-0140-3.

Additional file 1. Video of experiments. Video of experiments including the frequency response, release of thefabricated microrobot and actuation of microrobot are summarized.

\section{Acknowledgements}

Bioresist was provided by Nissan Chemical Corporation. We thank David MacDonald, MSc, from Edanz Group (http://www.edanzediting.com/ac) for editing a draft of this manuscript.

\section{Authors' contributions}

TW and TH are equally contributed to this work. TW and TH performed conception and design of the study, collection of data, analysis and interpretation of data, drafting of the manuscript and critical revision of the manuscript for important intellectual content. YY performed design of the study and critical revision of the article for important intellectual content. All authors read and approved the final manuscript.

\section{Funding}

This research was supported by a Chuo University Personal Research Grant.

\section{Availability of data and materials \\ Not applicable.}

\section{Competing interests}

The authors declare that they have no competing interests.

\section{Author details}

${ }^{1}$ Department of Precision Engineering, Chuo University, 1-13-27 Kasuga, Bunkyo-ku, Tokyo 112-8551, Japan. ${ }^{2}$ Toyama Industrial Technology Research and Development Center, 383 Takata, Toyama-shi, Toyama 930-0866, Japan.

Received: 14 July 2019 Accepted: 5 September 2019

Published online: 18 September 2019

\section{References}

1. Kamamichi N, Yamakita M, Asaka K, Luo Z-W (2006) A snake-like swimming robot using ipmc actuator/sensor. In: Proceedings 2006 IEEE international conference on robotics and automation, 2006. ICRA 2006. IEEE, New York, pp 1812-1817

2. Takane E, Tadakuma K, Yamamoto T, Konyo M, Tadokoro S (2016) A mechanical approach to realize reflexive omnidirectional bending motion for pneumatic continuum robots. ROBOMECH J 3(1):28

3. Takashima K, Kamizono H, Takenaka M, Mukai T (2017) Force sensor utilizing stiffness change of shape-memory polymer based on temperature. ROBOMECH J 4(1):17

4. Kurumaya S, Suzumori K, Nabae H, Wakimoto S (2016) Musculoskeletal lower-limb robot driven by multifilament muscles. ROBOMECH J 3(1):18

5. Niiyama R, Nagakubo A, Kuniyoshi Y. Mowgli (2007) A bipedal jumping and landing robot with an artificial musculoskeletal system. In: Proceedings 2007 IEEE international conference on robotics and automation. IEEE, New York, pp 2546-2551 
6. Marchese AD, Onal CD, Rus D (2014) Autonomous soft robotic fish capable of escape maneuvers using fluidic elastomer actuators. Soft Robot 1(1):75-87

7. Omori H, Hayakawa T, Nakamura T (2008) Locomotion and turning patterns of a peristaltic crawling earthworm robot composed of flexible units. In: 2008 IEEE/RSJ international conference on intelligent robots and systems. IEEE, New York, pp 1630-1635

8. Inose H, Yokoyama K, Imamura H, Kikutani I, Nakamura T (2015) Development of an end skeleton type power assist suit using pneumatic artificial muscles with amplification mechanism. In: IECON 2015-41st annual conference of the IEEE industrial electronics society. IEEE, New York, pp 004708-004713

9. Ilievski F, Mazzeo AD, Shepherd RF, Chen X, Whitesides GM (2011) Soft robotics for chemists. Angew Chem Int Ed 50(8):1890-1895

10. Hu W, Lum GZ, Mastrangeli M, Sitti M (2018) Small-scale soft-bodied robot with multimodal locomotion. Nature 554(7690):81

11. Yim S, Sitti M (2011) Design and rolling locomotion of a magnetically actuated soft capsule endoscope. IEEE Trans Robot 28(1):183-194

12. Jeon S, Hoshiar AK, Kim K, Lee S, Kim E, Lee S, Kim J-Y, Nelson BJ, Cha $\mathrm{H}-J$, Yi B-J et al (2019) A magnetically controlled soft microrobot steering a guidewire in a three-dimensional phantom vascular network. Soft Robot 6(1):54-68

13. Park S-J, Gazzola M, Park KS, Park S, Di Santo V, Blevins EL, Lind JU, Campbell PH, Dauth S, Capulli AK et al (2016) Phototactic guidance of a tissue-engineered soft-robotic ray. Science 353(6295):158-162

14. El-Etriby AE, Klingner A, Tabak AF, Khalil IS (2018) Manipulation of non-magnetic microbeads using soft microrobotic sperm. In: 2018 International conference on manipulation, automation and robotics at small scales (MARSS). IEEE, New York, pp 1-4

15. Hines L, Petersen K, Lum GZ, Sitti M (2017) Soft actuators for smallscale robotics. Adv Mater 29(13):1603483

16. Palagi S, Mark AG, Reigh SY, Melde K, Qiu T, Zeng H, Parmeggiani C, Martella D, Sanchez-Castillo A, Kapernaum N et al (2016) Structured light enables biomimetic swimming and versatile locomotion of photoresponsive soft microrobots. Nat Mater 15(6):647

17. Nawroth JC, Lee H, Feinberg AW, Ripplinger CM, McCain ML, Grosberg A, Dabiri JO, Parker KK (2012) A tissue-engineered jellyfish with biomimetic propulsion. Nat Biotechnol 30(8):792

18. Akiyama Y, Odaira K, Sakiyama K, Hoshino T, Iwabuchi K, Morishima K (2012) Rapidly-moving insect muscle-powered microrobot and its chemical acceleration. Biomed Microdevices 14(6):979-986
19. Yokoyama Y, Umezaki M, Kishimura T, Tamiya E, Takamura Y (2011) Micro-and nano-fabrication of stimulus-responsive polymer using nanoimprint lithography. J Photopolym Sci Technol 24(1):63-70

20. Hayakawa T, Kikukawa M, Maruyama H, Arai F (2016) Laser-driven gel microtool for single-cell manipulation based on temperature control with a photothermal conversion material. Appl Phys Lett 109(25):254102

21. Ichikawa A, Arai F, Yoshikawa K, Uchida T, Fukuda T (2005) In situ formation of a gel microbead for indirect laser micromanipulation of microorganisms. Appl Phys Lett 87(19):191108

22. Arai F, Ng C, Maruyama H, Ichikawa A, El-Shimy H, Fukuda T (2005) On chip single-cell separation and immobilization using optical tweezers and thermosensitive hydrogel. Lab Chip 5(12):1399-1403

23. Linder V, Gates BD, Ryan D, Parviz BA, Whitesides GM (2005) Water-soluble sacrificial layers for surface micromachining. Small 1(7):730-736

24. Tseng P, Pushkarsky I, Di Carlo D (2014) Metallization and biopatterning on ultra-flexible substrates via dextran sacrificial layers. PLoS ONE 9(8):106091

25. Hayakawa T, Sakuma S, Fukuhara T, Yokoyama Y, Arai F (2014) A single cell extraction chip using vibration-induced whirling flow and a thermoresponsive gel pattern. Micromachines 5(3):681-696

26. Arai F, Ichikawa A, Fukuda T, Katsuragi T (2003) Isolation and extraction of target microbes using thermal sol-gel transformation. Analyst 128(6):547-551

27. Ito K, Sakuma S, Yokoyama Y, Arai F (2014) On-chip gel-valve using photoprocessable thermoresponsive gel. ROBOMECH J 1(1):5

28. Loomis J, Fan X, Khosravi F, Xu P, Fletcher M, Cohn RW, Panchapakesan B (2013) Graphene/elastomer composite-based photo-thermal nanopositioners. Sci Rep 3:1900

29. Lu S, Ahir S, Velasco V, King B, Xu P, Terentjev EM, Panchapakesan B (2009) Photo-mechanical actuation of carbon nanotubes: mechanisms and applications in micro and nano-devices. J Micro-Nano Mechatron 5(1-2):29

30. Jiang W, Niu D, Liu H, Wang C, Zhao T, Yin L, Shi Y, Chen B, Ding Y, Lu B (2014) Photoresponsive soft-robotic platform biomimetic fabrication and remote actuation. Adv Funct Mater 24(48):7598-7604

31. Chen Y-W, Chen P-J, Hu S-H, Chen I-W, Chen S-Y (2014) Nir-triggered synergic photo-chemothermal therapy delivered by reduced graphene oxide/carbon/mesoporous silica nanocookies. Adv Funct Mater 24(4):451-459

\section{Publisher's Note}

Springer Nature remains neutral with regard to jurisdictional claims in published maps and institutional affiliations.

\section{Submit your manuscript to a SpringerOpen ${ }^{\circ}$ journal and benefit from:}

- Convenient online submission

- Rigorous peer review

- Open access: articles freely available online

- High visibility within the field

Retaining the copyright to your article

Submit your next manuscript at springeropen.com 